\title{
Evaluation of Performance of the Newly Developed de Cordova's Formula for Calculation of Low-Density Lipoprotein Cholesterol without Use of Triglycerides
}

\author{
Abdul Hai Siddique ${ }^{1}$, Muhammad Saiedullah ${ }^{2}$, Nasreen Chowdhury ${ }^{3}$, Md. Aminul Haque Khan ${ }^{4}$ \\ Received: July 22, 2013 Accepted: November 2, 2013
}

\begin{abstract}
Background: Various formulas are available to estimate serum low-density lipoprotein (LDL) cholesterol. All of these are serum triglycerides (TG) dependent. But very recently de Cordova et al developed a simple formula (CF) to calculate LDL cholesterol without using serum TG and claimed it to be more accurate than Friedewald's formula $(F F)$. Objective: The objective of the present study was to evaluate the performance of the CF for the calculation of $L D L$ cholesterol in a Bangladeshi population. Materials and Methods: Three hundred and sixty adult Bangladeshi subjects were purposively included in this study. Serum total cholesterol (TC), TG, high-density lipoprotein (HDL) cholesterol and LDL cholesterol were measured by direct automated methods. LDL cholesterol was also calculated by CF and FF. Results were expressed in conventional unit as mean $\pm S D$ and compared by two-tailed paired t test, bias against measured LDL cholesterol, Pearson's correlation coefficient (r), Passing \& Bablok regression and accuracy within $\pm 10 \%$ of the measured LDL cholesterol. Results: The mean values of directly measured LDL cholesterol, LDL cholesterol calculated by CF and FF were $117.7 \pm 31.0$, $111.8 \pm 31.0$ and $108.9 \pm 39.7 \mathrm{mg} / \mathrm{dL}$ respectively. Bias of calculated $L D L$ cholesterol against measured LDL cholesterol was $-5.2 \%$ for CF and $-9.6 \%$ for FF. The correlation coefficients of measured LDL cholesterol were $0.9796(p<0.001)$ for $C F$ and 0.9525 ( $p<0.001)$ for FF. Passing \& Bablok regression yielded the equation $y=0.9938 x-6.2$ for $C F$ and $y=1.2774 x-40.9$ for FF. Accuracy within $\pm 10 \%$ of measured LDL cholesterol was $81 \%$ for CF and 49\% for FF. Conclusion: This study revealed better performance of the de Cordova's formula than Friedewald's formula for approximate calculation of $L D L$ cholesterol without using serum triglycerides.
\end{abstract}

Key words: Low-density lipoprotein cholesterol, Calculated LDL cholesterol, Friedewald's formula, de Cordova's formula

J Enam Med Col 2014; 4(1): 10-14

\section{Introduction}

Serum low-density lipoprotein (LDL) cholesterol concentration is positively related to the incidence of coronary heart disease (CHD) $)^{1,2}$ and increased level of serum LDL cholesterol strongly correlates with the extent of atherosclerotic lesions. ${ }^{3}$ Lowering of LDL cholesterol decreases the incidence of major cardiovascular events. ${ }^{4,5}$ Therefore, measurement of

LDL cholesterol is crucial for the prevention and management of cardiovascular diseases (CVD). The reference method for the measurement of serum LDL cholesterol is the preparative ultracentrifugation, i.e., $\beta$-quantification. The ultracentrifugation method is time-consuming, costly, labor-intensive and requires expensive ultracentrifuges, rotors and

1. Research fellow, Department of Applied Laboratory Sciences, Bangladesh University of Health Sciences (BUHS), Dhaka

2. Assistant Professor, Department of Applied Laboratory Sciences, Bangladesh University of Health Sciences (BUHS), Dhaka

3. Professor, Department of Biochemistry, Chattagram Maa-O-Shishu Hospital Medical College, Chittagong

4. Professor, Department of Biochemistry, Enam Medical College, Savar, Dhaka

Correspondence Muhammad Saiedullah,Email:md.saiedullah@gmail.com 
tubes and only a few number of specimens can be investigated a day. Hence, its use in clinical practice is limited. ${ }^{6}$ To overcome these problems, several direct methods have been developed to measure serum LDL cholesterol. But all are expensive and not suitable for developing countries like ours.

Several formulas ${ }^{6-9}$ have been developed and used to calculate LDL cholesterol. Among these, Friedewald's formula $(\mathrm{FF})^{7}$ is used worldwide to estimate LDL cholesterol in routine clinical practice. All these formulas require the measurement of serum total cholesterol (TC), serum triglycerides (TG) and highdensity lipoprotein (HDL) cholesterol at fasting condition and are limited to fasting serum TG concentration up to $400 \mathrm{mg} / \mathrm{dL}$.

Studies in Bangladeshi population reported a remarkable underestimation of LDL cholesterol by Friedewald's formula and Anandaraja's formula. ${ }^{10-14}$ de Cordova et a ${ }^{15}$ recently published a new, simpler and less expensive formula (LDL cholesterol = $0.7516 \times$ [total cholesterol - HDL cholesterol]) independent of serum TG by analyzing lipid profiles of a large cohort of Brazilian population in fasting condition. It opens a new door to calculate LDL cholesterol in nonfasting state, since serum TG concentrations differ significantly and serum TC and HDL cholesterol concentrations differ minimally $(<5 \%)$ between fasting and nonfasting states. ${ }^{16,17}$ The aim of this study was to evaluate the performance of de Cordova's formula (CF) in a Bangladeshi population.

\section{Materials and Methods}

This cross-sectional study was conducted in the department of Applied Laboratory Sciences, Bangladesh University of Health Sciences (BUHS), Dhaka, Bangladesh during the period of January to June 2013. Venous blood specimens were collected for lipid analysis from the outpatient department of Bangladesh Institute of Health Sciences (BIHS) General Hospital from adult Bangladeshi subjects after 12 hours fast. After centrifugation, serum was separated and analyzed. Each specimen was preserved at $-80^{\circ} \mathrm{C}$ for the measurement of LDL cholesterol directly. All lipid parameters were measured by Dimension RxL Max autoanalyzer (Siemens Healthcare Diagnostics, Inc., USA). All kits, calibrators and quality control materials were obtained from Siemens Healthcare Diagnostics, Inc., USA through local distributor. LDL cholesterol was also calculated by de Cordova's formula and Friedewald's formula.

Blood specimens were collected purposively from 400 adult subjects. Forty subjects were excluded due to high serum TG concentration $(>400 \mathrm{mg} / \mathrm{dL})$ and the remaining 360 were considered as study subjects $(\mathrm{TG} \leqslant 400 \mathrm{mg} / \mathrm{dL}$ ). Results of the estimated LDL cholesterol were compared with the measured LDL cholesterol. For comparison, two-tailed paired $t$ test, Pearson's correlation coefficient and Bland-Altman plots $^{18-20}$ against measured LDL cholesterol and Passing \& Bablok $^{21}$ regression were applied. Accuracy of the estimated LDL cholesterol within $\pm 10 \%$ of measured LDL cholesterol was also determined. For statistical analysis $\mathrm{MedCalc}^{\circledR}$ version 11.4 for Windows was used.

\section{Results}

A total of 360 lipid profiles were included in this study. The mean age of the study subjects was $49 \pm$ 14 years. Among them 216 (60\%) were males and $144(40 \%)$ were females. The means of TC, TG, HDL cholesterol and measured LDL cholesterol were $188 \pm 44 \mathrm{mg} / \mathrm{dL}, 199 \pm 76 \mathrm{mg} / \mathrm{dL}, 39.0 \pm 8.2$ $\mathrm{mg} / \mathrm{dL}$ and $117.7 \pm 31.0 \mathrm{mg} / \mathrm{dL}$ respectively. The mean values of LDL cholesterol calculated by CF and FF were $111.8 \pm 31.0$ and $108.9 \pm 39.7 \mathrm{mg} / \mathrm{dL}$ respectively. Compared to measured LDL cholesterol, de Cordova's LDL cholesterol was $6.0 \pm 6.3$ $\mathrm{mg} / \mathrm{dL}$ lower $(p<0.001)$. The mean value of Friedewald's LDL cholesterol was $8.9 \pm 13.9 \mathrm{mg} / \mathrm{dL}$ lower $(p<0.001)$ than measured LDL cholesterol. Fig 1 shows the Bland-Altman plots of de Cordova's LDL cholesterol and Friedewald's LDL cholesterol against measured LDL cholesterol. The bias was $-5.2 \%$ for $\mathrm{CF}$ and $-9.6 \%$ for $\mathrm{FF}$.

The correlation coefficients of de Cordova's LDL cholesterol and Friedewald's LDL cholesterol were $0.9796(p<0.001)$ and $0.9525(p<0.001)$ respectively. Fig 2 shows the Passing \& Bablok regression of de Cordova's LDL cholesterol and Friedewald's LDL cholesterol against measured LDL cholesterol. de Cordova's LDL cholesterol yielded the regression equation $y=0.9938 x-6.2$ and Friedewald's LDL cholesterol yielded the regression equation, $y=$ $1.2774 x-40.9$ when calculated LDL cholesterol 
concentrations were plotted in $y$-axis against measured LDL cholesterol plotted in $x$-axis.
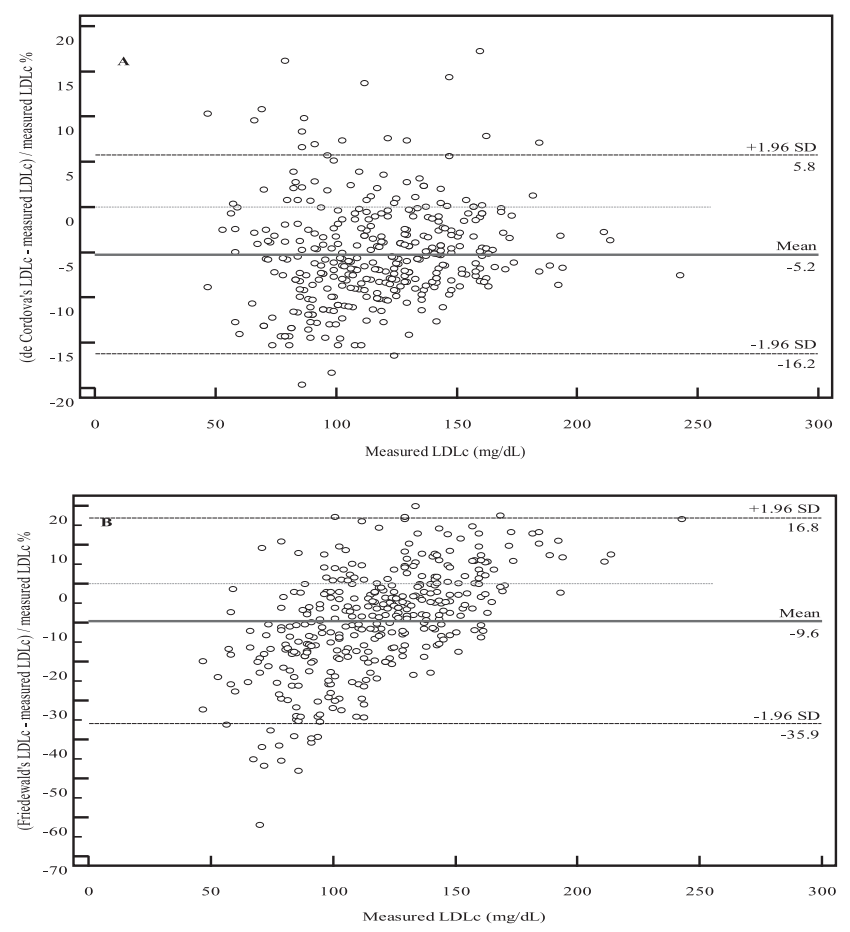

Fig 1. Bland-Altman plots of de Cordova's LDL cholesterol (A) and Friedewald's LDL cholesterol (B) against measured LDL cholesterol
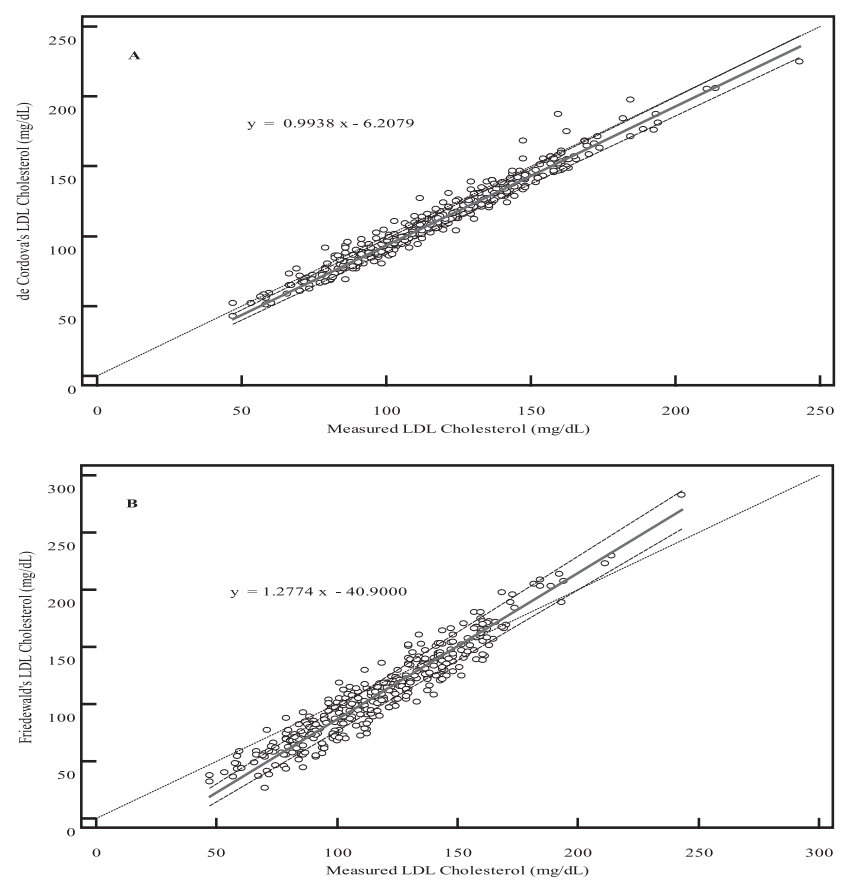

Fig 2. Passing \& Bablok regression of de Cordova's LDL cholesterol (A) and Friedewald's LDL cholesterol against measured LDL cholesterol
When percentages of differences are computed against measured LDL cholesterol, 291 (81\%) of de Cordova's LDL cholesterol fall within $\pm 10 \%$ of measured LDL cholesterol. On the other hand, 175 $(49 \%)$ of Friedewald's LDL cholesterol fall within $\pm 10 \%$ of measured LDL cholesterol.

The correlation coefficients of measured LDL cholesterol with TC, TG and HDL cholesterol were $0.962(\mathrm{p}<0.001), 0.356(\mathrm{p}<0.001)$ and 0.216 $(\mathrm{p}<0.001)$ respectively. Univariate linear regression analysis considering measured LDL cholesterol as dependent variable and non-HDL cholesterol as independent variable showed that the coefficient of non-HDL cholesterol was 0.7541 for measured LDL cholesterol $(\beta=0.974, \mathrm{p}<0.001)$.

\section{Discussion}

The correlation coefficient of LDL cholesterol calculated by the de Cordova's formula with the measured LDL cholesterol was statistically highly significant and better than the correlation coefficient of Friedewald's LDL cholesterol with measured LDL cholesterol ( $\mathrm{r}=0.9796$ vs $0.9525, \mathrm{p}<0.001)$. Though mean values of both de Cordova's LDL cholesterol and Friedewald's LDL cholesterol differed significantly from measured LDL cholesterol, mean difference and bias against measured LDL cholesterol were lower for de Cordova's LDL cholesterol compared to that of Friedewald's LDL cholesterol ( $-5.2 \%$ vs $-9.6 \%)$. Accuracy of de Cordova's formula within $\pm 10 \%$ of measured LDL cholesterol was also higher than Friedewald's formula $(81 \%$ vs $49 \%)$ and it was closer to the proportion $(87 \%)$ obtained by the regression equation ${ }^{10}$ developed for this population (data not shown). Passing \& Bablok regression equation clearly indicates the better performance of the de Cordova's formula compared to Friedewald's formula.

Studies done on Bangladeshi population in different settings showed remarkable underestimation of LDL cholesterol by Friedewald's formula. ${ }^{10-13}$ In this study the ratio of LDL cholesterol to non-HDL cholesterol was 0.7541 which is very similar to that of the de Cordova's study $(0.7516)^{15}$ (data not shown). Moreover, measured LDL cholesterol showed stronger relationship with TC $(\mathrm{r}=0.962$, $\mathrm{p}<0.001)$ compared to the relationship of measured 
LDL cholesterol with Friedewald's LDL cholesterol $(\mathrm{r}=0.940, \mathrm{p}<0.001)$. This result indicates that lower performance of Friedewald's formula may be associated with the variability of TG.

Most of the common formulas used the measurement of serum TC, serum HDL cholesterol and serum TG to estimate LDL cholesterol and requires the lipid measurement at fasting state. ${ }^{6-10}$ This limitation is due to inclusion of serum TG which significantly differs between fasting and nonfasting states. ${ }^{10,11}$ The others (TC, HDL cholesterol and LDL cholesterol) remain within acceptable range in fasting and nonfasting states. ${ }^{16,17}$ Hence, LDL cholesterol should be measured by direct method if lipid profiles are needed to be measured at nonfasting state. But most of the third world countries have no such ability. Since the de Cordova's formula has performance close to direct method and better performance than Friedewald's formula and is independent of serum TG and produces similar accuracy as the regression equation ${ }^{10}$ developed for this population, it may be helpful for the approximate calculation of LDL cholesterol at fasting and nonfasting states. However, more studies are recommended regarding its use in the calculation of LDL cholesterol in both fasting and nonfasting states in our population.

\section{References}

1. Gordon T, Kennel WD, Castelli WP, Dauber TR. Lipoproteins, cardiovascular disease and death: the Framingham Study. Arch Intern Med 1981; 141: $1128-1131$.

2. Anderson KM, Castelli WP, Levy D. Cholesterol and mortality: 30 years of follow-up from the Framingham Study. J Am Med Assoc 1987; 257: 2176-2180.

3. Newman WP, Freedman DS, Voors AW, Gard PD, Srinivasan SR, Cresanta JL et al. Relation of serum lipoprotein levels and systolic blood pressure to early atherosclerosis. The Bogalusa Heart Study. N Eng J Med 1986; 314: 138-144.

4. Lipid research clinics program: the lipid research clinics coronary primary prevention trial results: II. The relationship of reduction in incidence of coronary heart disease to cholesterol lowering. J Am Med Assoc 1984; 251: 365-374.

5. Heart Protection Study Collaborative Group. MRC/BHF heart protection study of cholesterol lowering with simvastatin in 20,536 high-risk individuals: a randomised placebo-controlled trial. Lancet 2002; 360: 7-22.

6. Rao A, Parker AH, EI-Sheronl NA, Babelly MM. Calculation of low-density lipoprotein cholesterol with the use of triglyceride/cholesterol ratios in lipoproteins compared with other calculation methods. Clin Chem 1988; 34: 2532-2534.

7. Friedewald WT, Levy RI, Fredrickson DS. Estimation of the concentration of low-density lipoprotein cholesterol in plasma, without use of the preparative ultracentrifuge. Clin Chem 1972; 18: 499-502.

8. DeLong DM, DeLong ER, Wood PD, Lipple K, Rifkind BM. A comparison of methods for the estimation of plasma low-density and very low-density lipoprotein cholesterol. The Lipid Research Clinics Prevalence Study. J Am Med Assoc 1986; 256: 2372-2377.

9. Hattori Y, Suzuki M, Tsushima M, Yoshida M, Toknaga Y, Wang Y et al. Development of approximate formula for LDL-chol, LDL-apoB and LDL-chol/LDL-apoB as indices of hyperapobetalipoproteinemia and small dense LDL. Atherosclerosis 1998; 138: 289-299.

10. Saiedullah M, Chowdhury N, Khan MAH, Hayat S, Begum S, Rahman MR. Comparison of regression equation and Friedewald's formula with direct measurement of low-density lipoprotein cholesterol in Bangladeshi population. J Sci Res 2014; 6: 143-152.

11. Kamal AHM, Hossain M, Chowdhury S, Mahmud NU. A comparison of calculated with direct measurement of low density lipoprotein cholesterol level. Journal of Chittagong Medical College Teachers' Association 2009; 20: 19-23.

12. Saiedullah M, Sarkar A, Kamaluddin SM, Begum S, Hayat S, Rahman MR et al. Friedewald's formula is applicable up to serum triacylglycerol to total cholesterol ratio of two in Bangladeshi population. Anwer Khan Modern Medical College Journal 2011; 2: 21-25.

13. Parvin M, Saiedullah M, Khan MAH, Rahman MR, Islam MS. Validation of the modified Friedewald's formula to calculate low-density lipoprotein cholesterol in Bangladeshi population. J Bangladesh Coll Phys Surg 2012; 30: 141-144.

14. Rahman MR, Saiedullah M, Shermin S, Ferdoush M. Comparison of low-density lipoprotein cholesterol calculated by Friedewald's formula and Anandaraja's formula in a Bangladeshi population. Bang $\mathrm{J}$ Med Biochem 2009; 2: 18-20.

15. de Cordova CMM, de Cordova MM. A new accurate, simple formula for LDL-cholesterol estimation based on directly measured blood lipids from a large cohort. Ann Clin Biochem 2013; 50: 13-19. 
16. Mora S, Rifai N, Buring JE, Ridker PM. Fasting compared with nonfasting lipids and apolipoproteins for predicting incident of cardiovascular events. Circulation 2008; 118: 993-1001.

17. Langsted A, Freiberg JJ, Nordestgaard BG. Fasting and nonfasting lipid levels: influence of normal food intake on lipids, lipoproteins, apolipoproteins and cardiovascular risk prediction. Circulation 2008; 118: 2047-2056.

18. Bland JM, Altman DG. Statistical methods for assessing agreement between two methods of clinical measurement. Lancet 1986; 327: 307-310.

19. Bland JM, Altman DG. Measuring agreement in method comparison studies. Stat Methods Med Res 1999; 8: $135-160$

20. Krouwer JS. Why Bland-Altman plots should use X, not $(\mathrm{Y}+\mathrm{X}) / 2$ when $\mathrm{X}$ is a reference method. Statistics in Medicine 2008; 27: 778-780.

21. Passing H, Bablok W. A new biometrical procedure for testing the equality of measurements from two different analytical methods. Application of linear regression procedures for method comparison studies in clinical chemistry, part I. J Clin Chem Clin Biochem 1983; 21: 709-720. 